\title{
La Mortalite Infanto-Juvenile A Bouafle : Contribution A L'analyse Des Conditions De Vie Dans Une Ville Ivoirienne
}

\author{
Kadet G. Bertin \\ Enseignant-chercheur à l'Ecole Normale Supérieure d'Abidjan, \\ Côte d'Ivoire \\ Adjelou Kessou \\ Doctorant en géographie, \\ Université Jean Lorougnon Guédé de Daloa, Côte d'Ivoire \\ Anoh Kouassi Paul \\ Professeur titulaire de géographie, \\ Université Félix Houphouët-Boigny, Abidjan, Côte d'Ivoire
}

Doi: 10.19044/esj.2018.v14n2p48 URL:http://dx.doi.org/10.19044/esj.2018.v14n2p48

\begin{abstract}
The dynamics of Bouaflé, an urban locality in the center-west of Côte d'Ivoire, faces a double challenge. On the one hand, the plight related to the mortality of the under-five stands out as a threat to their future. On the other hand, owing to lack of drinking water in the city as well as harmful effects of polluting activities on the local environment, environmental pathologies affect the urban space. From available documents on this city supplemented by field surveys, this study showcases an inventory of the determining factors of infant and child mortality in Bouaflé and highlights the limitations of local governance in the quest for a sustainable development for the capital city of the Marahoué.
\end{abstract}

Keywords: Bouaflé, infant and child mortality, environmental pathologies, determining factors, sustainable development, local governance

Résumé

La dynamique de Bouaflé, une localité urbaine située dans le CentreOuest ivoirien, est confrontée à un double défi. D'une part, l'importance de la mortalité infanto- juvénile qui frappe les enfants de moins de cinq ans, représente une menace pour le futur de ces enfants. D'autre part, des pathologies environnementales affectent l'espace urbain, dues à un déficit d'alimentation de la ville en eau potable, ainsi que des effets néfastes 
d'activités polluantes sur l'environnement local. A partir des documents disponibles concernant cette ville, et grâce à des enquêtes de terrain, la présente étude dresse un inventaire des déterminants de la mortalité infantojuvénile à Bouaflé, et présente les limites de la gouvernance locale, dans la quête d'un développement durable de la capitale du Marahoué.

Mots clés : Bouaflé, mortalité infanto- juvénile, pathologies environnementales, déterminants, développement durable, gouvernance locale

\section{Introduction}

La Côte d'Ivoire figure parmi les pays ayant un niveau élevé de mortalité des enfants de moins de cinq ans. D'après un rapport de la Banque mondiale, le taux de mortalité infanto- juvénile a été successivement, 127\%o en $2006,118,5 \%$ en $2009,123 \%$ en 2010 et de $115 \%$ en 2011 dans ce pays (Banque Mondial, rapport 2011). Bien que les estimations du Groupe interagences des Nations-Unies (UNICEF, OMS, Banque mondiale, Département des affaires économiques et sociales de l'ONU, PNUD) y notent une évolution en baisse de la mortalité infanto-juvénile, les taux étant de 108\%o en 2012, $99 \%$ en $2013,96 \%$ en 2014 et $93 \%$ en 2015 , cette tendance présente des inégalités. C'est ce que révèle la classification de l'Institut National de Statistique (INS), indiquant que les taux les plus bas dans le pays sont inférieurs à $62 \%$, et les plus élevés, supérieurs à $92 \%$. Ces estimations sont la preuve qu'en Côte d'Ivoire, l'indice de santé reste préoccupant. Dans un contexte de développement durable des implantations socio-spatiales, la situation sanitaire de ce pays inquiète, et interpelle la gouvernance urbaine, notamment à Bouaflé, chef-lieu de la Région de Marahoué, dans le CentreOuest ivoirien.

Cette ville située dans une zone de transition, entre la forêt et la savane arborée, fait partie des villes ivoiriennes présentant ce pic national (92\%) de mortalité infanto- juvénile (INS, 2012). La ville est traversée par un affluent du fleuve Bandaman, la Marahoué dont le cours est investi par d'intenses activités économiques et industrielles dans ses environs immédiats. Une soixantaine de kilomètres de voies bitumées la séparent de Yamoussoukro, la capitale administrative du pays, dont elle subit les influences de proximité, grâce aux activités d'une population évaluée à 85394 habitants, et composée d'autochtones Gouro, Ayaou, Yaouré, d'allogènes et de non Ivoiriens (RGPH 2014). Pour les besoins sanitaires de cette population, l'Etat y a développé d'importantes infrastructures, notamment un Centre Hospitalier Régional (CHR), un centre de Protection Maternelle et Infantile (PMI), une maternité dotée d'un service de pédiatrie. Ainsi, une attention particulière est portée aux 
enfants de moins de cinq ans qui bénéficient, également, de la gratuité des consultations, de produits pharmaceutiques, et de campagnes de vaccination.

En dépit de ces dispositions, le taux de mortalité infanto-juvénile de la ville demeure l'un des plus élevés du pays. Or, d'après les Nations Unies, plus de 2 millions de personnes, pour la plupart des jeunes, meurent chaque année, suite à des maladies causées par des eaux sales et l'absence de sanitaires. Du fait de ce constat macabre, l'organisation supranationale a alors proclamé en 2000, comme un droit de l'homme, l'accès à l'eau potable et à des installations sanitaires, et fait de l'assainissement, un des Objectifs du Millénaire pour le Développement, en 2008.

Compte tenu de l'intérêt de la communauté internationale pour un développement durable des villes, la situation sanitaire à Bouaflé mérite que l'on analyse les déterminants de la mortalité infanto- juvénile affectant cette localité. Pourquoi, la mortalité infanto-juvénile à Bouaflé est-elle parmi les plus élevés en Côte d'Ivoire? Quelle est l'impact des facteurs environnementaux sur la situation sanitaire de cette ville, pourtant située non loin de Yamoussoukro, capitale politique et vitrine du pays ? Quel est le rôle de la gouvernance locale, en vue d'améliorer les conditions de la vie citadine ?

\section{Methode et outils}

Quatre procédures de collecte des informations sont observées pour réaliser cette étude, notamment, la revue de la littérature, l'exploitation de documents administratifs, l'observation et l'enquête de terrain. Concernant l'enquête de terrain, deux méthodes d'échantillonnage ont été nécessaires pour le choix des quartiers et des populations enquêtées à Bouaflé. Compte tenu de l'étendue de la ville, du nombre important de quartiers et de la similitude de leurs caractéristiques, le sondage stratifié a été privilégié pour le choix des quartiers à enquêter. Dans cette perspective, les quartiers ont été répartis en sous-ensembles homogènes selon leurs caractéristiques, et un tirage au sort a été fait dans chaque sous-ensemble. Il est à noter que dans les différents sousensembles constitués, les quartiers les plus importants ont été choisis pour représenter chaque sous-ensemble. Les quartiers Dioulabougou, Déhita, Koko-commerce, Agbanou et le quartier Résidentiel ont été alors retenus (carte 1).

Concernant la population enquêtée, une liste des cibles a été produite en tenant compte de la composition des ménages. Toutefois, certains ménages n'ayant pas d'enfants de moins de cinq ans, c'est l'échantillonnage aléatoire simple qui a permis de déterminer un total de 416 
Carte 1 : Localisation des quartiers enquêtés



ménages enquêtés. Enfin, la méthode des quotas impose de respecter les répartitions afin de représenter les cinq quartiers, proportionnellement aux ménages. Au final, la population enquêtée se présente comme suit : 187 ménages à Dioulabougou, 100 à Déhita, 12 à Koko-Commerce, 96 à Agbanou et 21 au quartier Résidentiel.

Des informations complémentaires ont été obtenues auprès des agents travaillant dans les services publics de Bouaflé. Au nombre de ces services figurent l'unité de pédiatrie de la direction de la santé, la direction régionale de l'environnement et du développement durable, la direction régionale de l'hydraulique territoriale et la direction du service technique et du service d'hygiène de la mairie. Les données recueillies ont été analysées à partir de deux méthodes. La première, la méthode statistique, s'est appuyée sur le 
logiciel Excel pour l'organisation, la présentation des données, l'extraction d'informations et la recherche de liens entre les données. Puis la seconde, la méthode cartographique à travers le logiciel Arcgis, a permis d'élaborer la distribution, la répartition et l'intensité des données.

\section{Resultats}

\section{Des pathologies liees au milieu socio- environnemental : les facteurs de la} mortalite infanto- juvenile a bouafle,

- $\quad$ Un espace urbain affecté par des maladies endémiques

L'espace urbain local présente des pathologies entraînant des décès d'enfants dont l'âge oscille entre zéro et cinq ans. D'après les services de santé de la ville, les motifs de la mortalité y sont dus à l'anémie, la diarrhée, les douleurs abdominales, la fièvre typhoïde, la gastroentérite, l'ictère, l'infection néonatale, les maladies hémorragiques, la méningite, le paludisme, la pneumopathie, la prématurité, la souffrance cérébrale, le tétanos et les troubles métaboliques. Parmi ces maladies, sept sont régulièrement présentes lors des évaluations annuelles. Ces maladies touchent un grand nombre d'individus. Il s'agit de l'anémie, la diarrhée, l'infection néonatale, la méningite, le paludisme, la pneumopathie et les souffrances cérébrales. En cinq ans, ces maladies ont occasionné 555 décès sur 583 , soit $95,20 \%$ des cas de décès. Huit autres pathologies, survenant de façon irrégulière, sont responsables de moins de 5\% des décès dans la ville, soient 28 cas de décès sur 583, entre 2012 et 2016 (Tableau 1).

Tableau 1: Les maladies endémiques dans la mortalité infanto-juvénile

\begin{tabular}{|c|c|c|c|c|c|c|}
\hline Motifs de mortalité & 2012 & 2013 & 2014 & 2015 & $\begin{array}{c}\text { Jusqu'en juin } \\
2016\end{array}$ & Total \\
\hline Anémie & 11 & 3 & 10 & 11 & 2 & 37 \\
\hline Diarrhée & 3 & 1 & 1 & 2 & 3 & 10 \\
\hline Infection néonatale & 2 & 1 & 2 & 7 & 2 & 14 \\
\hline Méningite & 4 & 10 & 2 & 3 & 3 & 22 \\
\hline Paludisme & 50 & 86 & 112 & 97 & 26 & 371 \\
\hline Pneumopathie & 7 & 1 & 3 & 12 & 3 & 26 \\
\hline Souffrance cérébrale & 13 & 12 & 17 & 18 & 15 & 75 \\
\hline $\begin{array}{c}\text { Total des maladies } \\
\text { endémiques }\end{array}$ & 90 & 114 & 147 & 150 & 54 & 555 \\
\hline Mortalité totale & 100 & 117 & 154 & 153 & 59 & 583 \\
\hline $\begin{array}{c}\text { Proportion des } \\
\text { maladies endémiques }\end{array}$ & $90 \%$ & $97,44 \%$ & $95,46 \%$ & $98,04 \%$ & $91,53 \%$ & $95,20 \%$ \\
\hline Source Direction
\end{tabular}

Source : Direction de l'Information, de la Planification et de l'Evaluation (DIPE), Bouaflé

Au nombre de ces maladies, le paludisme détient $66,85 \%$ des cas de décès. Depuis 2012, l'influence de cette pathologie est très significative car, elle représente plus de la moitié des cas de mortalité, et des maladies endémiques de la ville. D'après les services de santé urbains, le paludisme a 
une incidence de $50 \%$ sur la mortalité infanto-juvénile de la ville, alors qu'entre 2013 et 2015, son impact a été considérable. Ainsi, elle a entraîné $73,5 \%$ des cas de décès en $2013,72,73 \%$ en 2014 et 63,4\% en 2015 . Viennent ensuite les souffrances cérébrales et l'anémie causant respectivement 13,51\% et $06,67 \%$ de cas de maladies.

- $\quad$ Une mortalité due aux maladies environnementales

Les facteurs physiques, chimiques, biologiques et socio-économiques dans lequels vivent les hommes, les animaux et les végétaux influencent la vie. Ces éléments de l'environnement sont interdépendants, si bien que leur interaction dans un milieu, a des conséquences sur leur vie. Ainsi, l'utilisation de l'eau, les déchets ménagers et les déchets des activités, ont un impact sur la santé des citadins.

A Bouaflé, la mortalité infanto-juvénile est provoquée par des maladies d'origine virale ou bactérienne. Ces virus et bactéries proviennent de l'environnement dans lequel vivent les enfants de moins de cinq ans. Ce sont les conditions naturelles, les conséquences de l'utilisation de la nature agissent sur les enfants. En effet, les maladies environnementales les plus fréquentes ici, sont le paludisme, les infections respiratoires aigües, les maladies diarrhéiques, la tuberculose, le pian, la varicelle, la conjonctivite, le choléra, la fièvre jaune et la fièvre typhoïde. Parmi elles, les maladies diarrhéiques, la fièvre typhoïde, le paludisme et la pneumopathie ont un impact sur la mortalité des enfants au niveau local.

Tableau 2: Maladies environnementales et cas de décès d'enfants (chiffres)

\begin{tabular}{|c|c|c|c|c|c|c|}
\hline $\begin{array}{c}\text { Années } \\
\text { Causes de- }\end{array}$ & 2012 & 2013 & 2014 & 2015 & 2016 & Total \\
\hline Maladies diarrhéiques & 5 & 4 & 1 & 2 & 3 & 15 \\
\hline Fièvre typhoïde & 2 & & 1 & 1 & 2 & 6 \\
\hline Paludisme & 50 & 86 & 112 & 97 & 26 & 371 \\
\hline Pneumopathie & 7 & 1 & 3 & 12 & 3 & 26 \\
\hline $\begin{array}{c}\text { Total des maladies liées à } \\
\text { l'environnement }\end{array}$ & 64 & 91 & 117 & 112 & 34 & 418 \\
\hline Total des décès d'enfants & 100 & 117 & 154 & 153 & 59 & 583 \\
\hline $\begin{array}{c}\text { Pourcentage de maladies } \\
\text { liées à l'environnement (\%) }\end{array}$ & $64 \%$ & $77,78 \%$ & $75,97 \%$ & $73,20 \%$ & $57,62 \%$ & $71,7 \%$ \\
\hline
\end{tabular}

Source : DIPE, Bouaflé

Entre 2012 et 2016, ces quatre maladies ont causé 418 décès d'enfants de moins de cinq ans sur 583 cas enregistrés, soit 71,7\% (Tableau 2). Elles maladies représentent les principales causes de décès depuis 2012, entraînant au moins $64 \%$ de la mortalité, avec un impact particulièrement significatif en 2013. Dans ce tableau, les maladies endémiques représentées par le paludisme, la pneumopathie et les maladies diarrhéiques ont causé les ravages les plus importants parmi les populations. Dans l'ordre d'importance destructrice, le 
paludisme occupe la première place, étant responsable de $88,76 \%$ des décès depuis cinq ans. Suivent ensuite la pneumopathie, les maladies diarrhéiques et la fièvre typhoïde, causant respectivement $6,22 \%, 3,59 \%$ et $1,44 \%$ des décès entre 2012 et 2016.

Cependant, les consultations médicales dans les hôpitaux de Bouaflé, ont permis de diagnostiquer diverses maladies responsables de la mortalité dans la ville. Ainsi, mi-juin 2016, 9 pathologies ont été mises en évidence. Déjà en 2012, 11 pathologies ont occasionné plusieurs décès d'enfants de moins de cinq ans, contre 8 en 2013, 12 en 2014 et 10 en 2015. A la fin de 2016, 22 maladies ont été diagnostiquées lors des consultations dans les principaux centres de santé de cette localité. Il s'agit notamment de l'anémie, la bilharziose urinaire, la conjonctivite, la coqueluche, la dermatose, les douleurs abdominales, la fièvre jaune, la fièvre typhoïde, l'ictère, les infections néonatales, les infections respiratoires, les maladies diarrhéiques, les maladies hémorragiques, la malnutrition, la méningite, la prématurité, le paludisme, le SIDA, les souffrances cérébrales, le tétanos, les troubles métaboliques et la tuberculose. Le rapport entre les décès et les consultations est résumé dans le tableau 3 ci- après :

Tableau 3 : Consultations médicales dans les centres de santé, et cas de décès d'enfants à Bouaflé (chiffres)

\begin{tabular}{|c|c|c|c|c|c|}
\hline Période & 2012 & 2013 & 2014 & 2015 & $\begin{array}{c}\text { mi- } \\
2016\end{array}$ \\
\hline Nombre de consultations & 42974 & 48269 & 47394 & 78698 & 31759 \\
\hline Nombre de décès & 100 & 117 & 154 & 153 & 56 \\
\hline
\end{tabular}

Source : DIPE, Bouaflé

En observant les motifs de la mortalité infantile et les maladies diagnostiquées lors des consultations médicales, l'on réalise que les centres de santé sont de plus en plus fréquentés par les patients. La dynamique de fréquentation des centres de santé révèle toutefois l'importance des maladies infantiles dans la ville. Cependant, depuis que les visites se multiplient dans les centres de santé, la proportion des décès diminue. Après la croissance constatée entre 2012 et 2014, la tendance est à une baisse de la mortalité, et ce, à partir de 2015 (Tableau 3). En effet, d'après les services médicaux locaux, sur 1000 consultations d'enfants de moins de cinq ans effectuées, 02 décès d'enfants sont enregistrés en moyenne par an, ce qui confirme la baisse, et témoigne également des efforts d'éradication effectués par lesdits services.

En outre, l'on note que, sur 22 motifs de consultation, 14 sont des causes de décès. Parmi ces dernières, 11 sont peu fréquentes à la consultation. Cependant, elles sont les plus ravageuses en termes de décès. A l'échelle de la ville, l'apparition des ces maladies, qui représentent la moitié des motifs de consultation, est synonyme de décès. Cette situation s'explique par l'importance relative accordée aux maladies les plus fréquemment 
diagnostiquées, notamment le paludisme, l'anémie ou la fièvre typhoïde. Ces dernières retiennent de ce fait, l'attention des services médicaux de soins. En conséquence, leur incidence sur la mortalité diminue.

Toutes ces pathologies identifiées sont présentes dans tous les quartiers de la ville. Notre analyse de la distribution des maladies régulièrement diagnostiquées dans les services urbains, révèle un paysage contrasté. Très clairement, certaines maladies se rencontrent dans toute la ville, alors que d'autres sont sectorielles. Les maladies communes à l'ensemble du centre urbain sont au nombre de six (la méningite, la souffrance cérébrale, l'anémie, la dermatose, la fièvre typhoïde et le paludisme), et elles sont liées à l'environnement (paludisme, fièvre typhoïde, dermatose) ou à des conditions de vie difficiles (méningite, souffrance cérébrale, anémie).

Quatre autres maladies sont régulièrement repérées dans quelques quartiers. Il s'agit des infections respiratoires, des maladies diarrhéiques, de la Bilharziose urinaire et de la conjonctivite. Les infections respiratoires sont présentes dans trois des seize quartiers (Koko-commerce, Dioulabougou, Déhita. Quant aux maladies diarrhéiques et la conjonctivite, elles impactent quatre quartiers, tandis que la bilharziose urinaire s'observe dans cinq quartiers (carte 2). Les quartiers Koko-commerce et Dioulabougou concentrent toutes les maladies.

Carte 2 : Les pathologies identifiées dans les quartiers de Bouaflé.

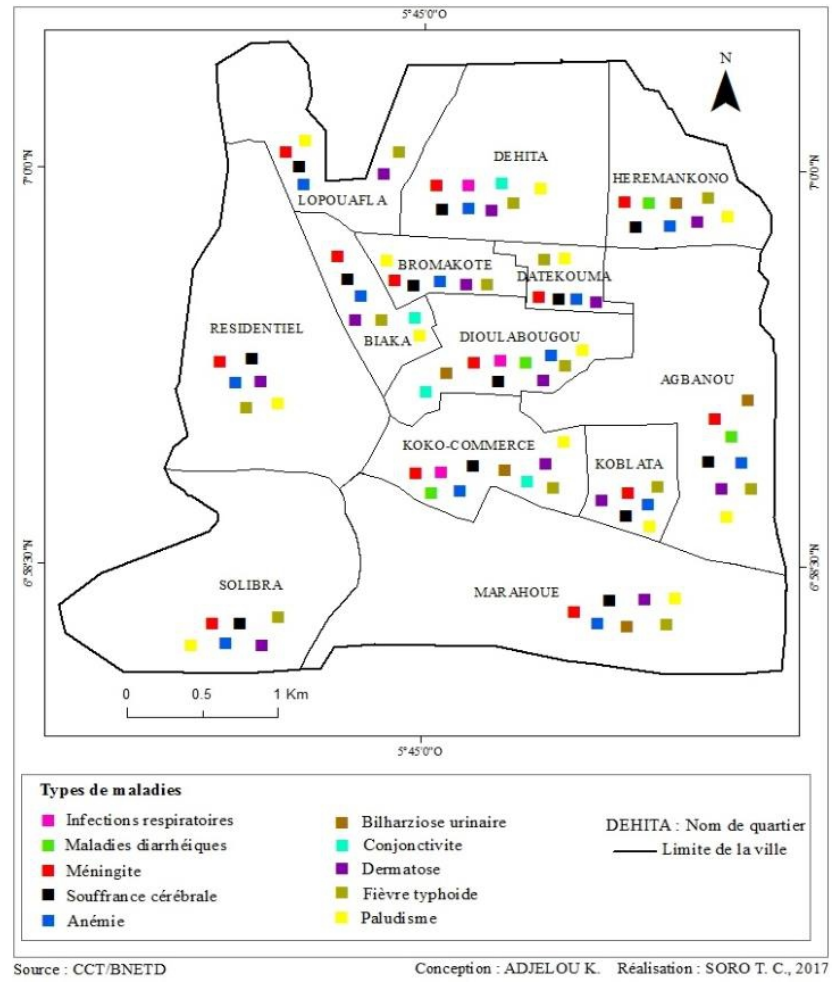




\section{Environnement et cadre de vie : le regne de l'insalubrite}

Des difficultés d'alimentation en eau potable

Depuis plusieurs années, la consommation urbaine d'eau à Bouaflé est estimée à $2.310 \mathrm{~m}^{3} /$ jour. Cette eau potable provient essentiellement des premières installations publiques destinées à couvrir les besoins de la ville. Bien qu'insuffisante pour toute la ville, le volume d'eau permettait d'alimenter la plupart des quartiers. Cependant, les effets de l'étalement urbain ont eu pour conséquence l'intégration de villages environnants dans les périmètres urbains. Dans cette dynamique, la localité rurale de Déhita, dans le Nord de Bouaflé, s'est agrandie au point d'être l'un des quartiers les plus étendus de la ville. C'est également le cas des anciens quartiers, Hermankono et Agbanou devenues de vastes espaces très peuplés. A l'échelle locale, il est de notoriété qu'Agbanou est le plus grand quartier de Bouaflé. Deux autres nouveaux quartiers, Marahoué et Port-Bouet bien peuplés s'ajoutent aux premiers quartiers.

L'extension du paysage urbain accroît les besoins en eau de la ville. Pour être autosuffisante, celle-ci a besoin de $4.000 \mathrm{~m}^{3} / \mathrm{jour}$, d'après les estimations de la Direction Territoriale de 1'Hydraulique de Bouaflé. Les extensions de quartiers et les nouveaux quartiers vivent dans la précarité, à cause du manque d'équipements collectifs, en particulier l'absence d'adduction d'eau potable. Or, ces extensions sont des zones de fortes concentrations de populations. 4694 ménages y ont été dénombrés dans, sur un total de 12672 ménages que compte la ville, soit 37,04\% des ménages urbains (RGPH, 2014).

Pourtant, Bouaflé est traversé par le fleuve Marahoué, un affluent du Bandama du plus grand fleuve ivoirien. La mobilisation des potentialités locales de cette ressource naturelle est loin d'être mise en valeur, contrairement à ce que proposent certains travaux, mettant en évidence le lien étroit existant entre le territoire et le développement local (Borda, 2016 : 261267). En conséquence, pour pouvoir s'approvisionner en eau potable, certains ménages ont recours à des raccordements anarchiques au réseau national, d'autres creusent des puits à l'intérieur des habitations. Cette situation inquiète, d'autant plus que le dernier Recensement Général de la Population et de l'Habitat (RGPH, 2014) atteste d'une forte augmentation de la population de Bouaflé, passé de 58.037 habitants en 1998 à 85.394 en 2014 (RGPH, 2014). Cette évolution de population urbaine n'est pas sans conséquence sur les besoins en eau potable des ménages. En effet, Bouaflé souffre d'une forte baisse de pression de l'eau. Les extensions urbaines et les nouveaux fronts d'urbanisation sont les plus exposés aux pénuries d'eau, dû à la baisse de pression. Pour ces quartiers éloignés des tuyaux à gros diamètre, la baisse de pression est devenue un fait banal, puisque vécue au quotidien. C'est le cas des quartiers Déhita et Port-Bouet. Pourtant, l'ancienne ossature de la ville est 
normalement alimentée en eau potable provenant des installations du réseau national de la SODECI.

L’insuffisance des équipements électriques et des adductions d'eau potable, obligent la population à s'agglomérer dans le périmètre de l'ancienne ossature urbaine. Cette partie de la ville rassemble 7978 ménages, soit $62,96 \%$ des ménages, dont la plupart sont affectées par la baisse de la pression d'eau.

Le manque d'eau dans les quartiers est dû à des difficultés de production, de stockage et de distribution de ce produit. En effet, concernant la production, la capacité de l'usine de traitement d'eau est dépassée, eu égard à l'évolution démographique dans les quartiers. A partir de l'eau de la Marahoué, l'usine traite et alimente la ville. Cependant, cette eau est insuffisante pour couvrir les besoins locaux. les quartiers périphériques, notamment les nouveaux fronts d'urbanisation peinent à être ravitaillés en eau potable. L'unique château d'eau de la ville situé à Koko-commerce, d'une capacité évaluée à $500 \mathrm{~m}^{3} /$ jour, ne suffit pas pour toute la ville. La nécessité d'une infrastructure de stockage et d'approvisionnement en eau potable d'une capacité supérieure s'impose à Bouaflé. Dans l'attente d'un nouveau château d'eau, les autorités municipales de la ville sont interpelées pour accroître le volume de l'existant, surtout dans un contexte de croissance démographique où la population de la ville a atteint 85394 habitants (RGPH, 2014). En outre, le diamètre des tuyaux alimentant le réseau de distribution interne étant trop réduit, une opération visant l'augmentation du volume de l'actuel château, devra inclure nécessairement, une adaptation du diamètre des tuyaux qui desservant les quartiers où l'adduction en eau potable est faible.

Finalement, les difficultés d'accès à l'eau potable à Bouaflé impactent la qualité de vie des populations, qui se dégrade dans la cité du Marahoué. Le manque d'eau potable dans la plupart des ménages urbains oblige ces derniers à utiliser des eaux non traitées, impropres à la consommation, ce qui est à l'origine de diverses maladies environnementales (supra). Cette situation est également liée à un déficit en matière d'assainissement car, les rues, les fosses septiques, les puits perdus, les caniveaux ouverts et les abords des habitations constituent des espaces de déjection ou d'évacuation de déchets ménagers. Seule la fosse septique est appropriée pour une évacuation hygiénique des eaux usées mais, dans la ville, les installations sanitaires améliorées sont rares. Cependant, le quartier Koko-Commerce dispose d'un réseau d'assainissement ayant des caniveaux recouverts de dalles pour faciliter le drainage des eaux usées vers les eaux de la rivière Marahoué. Partout ailleurs, les fosses septiques prolifèrent. Mis à part ces moyens d'évacuation, les eaux usées et les excréta se retrouvent en contact direct avec les enfants car, directement déversées dans l'environnement de la ville (Photo 1). Cette situation affecte les temporalités des espaces publics dans la ville, telles que rapportées par 
certains chercheurs (Guillemot, 2015 :105-141), faisant de Bouaflé, une ville où les promenades à l'air libre présentent des risques pour la santé des usagers.

Photo 1 : L'élimination des eaux usées au quartier Hermankono

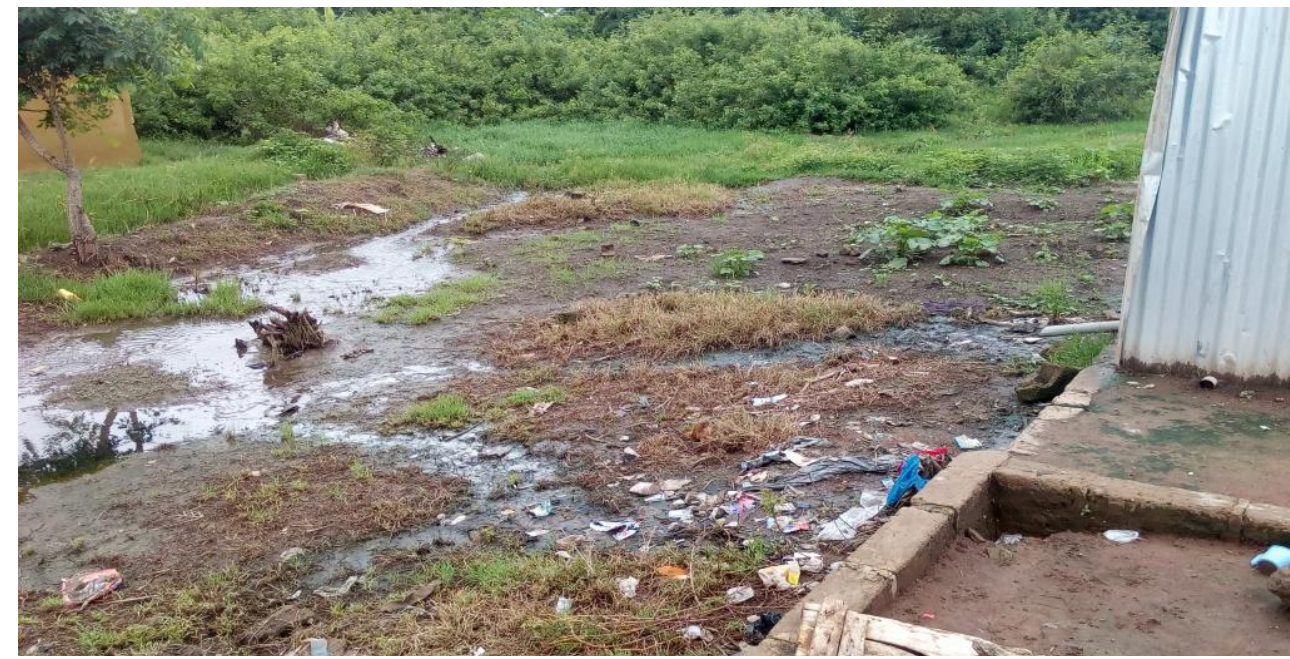

Cliché : ADJELOU Kessou, Bouaflé, décembre 2016

Au total, le paysage urbain local présente d'importantes étendues de déchets, principalement dans sept des seize quartiers de Bouaflé. La moitié de l'espace urbain baigne dans l'insalubrité. C'est le cas des quartiers Agbanou extension, Bromakoté, Datécouma, Déhita, Dioulabougou, Hermankono et Lopoufla, où résident 6231 ménages, soit 49,17\% des ménages urbains. Il en résulte que les habitants de ces quartiers, en particulier les enfants de moins de cinq ans sont, de fait, exposés à des risques de maladies. Toutefois, neuf autres quartiers bénéficiant d'un relatif aspect salubre, échappent au sort des premiers. Cette situation d'insalubrité avancée dans laquelle évolue la ville, est une conséquence des assainissements anarchiques initiés çà et là par des privés. Ces assainissements privés étant tournés vers la satisfaction des besoins de leurs auteurs, l'environnement extérieur des habitats est négligé, favorisant la multiplication d'étendues d'eaux usées, en l'absence d'un dispositif de canalisation cohérente et adaptée.

- $\quad$ Activités socio-économiques et environnement urbain

Plusieurs activités économiques concourent à la pollution de l'environnement, affectant l'image de la ville. Le commerce, les garages automobiles, certaines usines et les friches urbaines en sont quelques exemples. Le commerce est la deuxième activité la plus importante de Bouaflé, après l'agriculture, qui exerce un impact négatif sur l'environnement urbain. Partout dans les quartiers, de petits marchés improvisés offrent de nombreux endroits où sont entassés des restes de produits avariés. A côté de ces marchés, il y a des commerces de vivriers en plein sol (manioc et banane 
exclusivement), des commerces de banane mûres (alloco), de charbon, de gâteaux, de jus, etc. Ces commerces ne se font pas dans des locaux mais, en pleine rue et sur des installations de fortune. L'impact environnemental des restaurateurs et tenanciers de maquis est aussi très important, car ces activités sont très répandus à Koko-commerce. Une fois les marchés évacués en fin de journée, l'espace se transforme en une poubelle à ciel ouvert, jonchée de détritus.

De toutes ces activités, le commerce de l'attiéké, un produit à base de manioc, est le plus destructeur de l'environnement. L'espace urbain local est considérablement influencé par la transformation de ce tubercule. L'eau de manioc provient du pressage du manioc broyé et mis en pattes. Après conditionnement et séchage, le produit fini ou l'attiéké, est commercialisé à des fins de consommation. Il se trouve que le liquide issu du conditionnement du manioc est rejeté dans la nature sans précaution, alors que l'odeur qui s'en dégage, en plus des désagréments causés aux usagers, attire les moustiques et rendant l'environnement malsain. Par infiltration progressive, cette eau sale pollue le sol et la nappe phréatique. Le sol devient inapte à toute autre activité économique. Les épluchures de manioc abandonnées dans la nature, participent également de la pollution de l'environnement.

Pour pallier les problèmes de déchets liquides, les autorités municipales ont aménagé des espaces réservés à la transformation du manioc. C'est le cas au quartier Agbanou, où passe la rivière Gropéni dont les abords ont été aménagés pour accueillir les activités de transformation du manioc. L'eau issue du manioc est alors directement drainée vers la rivière. Dans certains secteurs du quartier, la rivière étant éloignée des habitations, des espaces sont prévus en plein quartier.

Les activités liées au transport, et impliquant les garages des automobiles et des motocyclistes participent à la dégradation de l'environnement. Elles jonchent les voies de communication des quartiers. Les mécaniciens déversent des huiles minérales usagées provenant des vidanges des moteurs, lesquelles laissent des impacts indélébiles sur les sites et sur les sols. A la faveur des pluies, les huiles inondent les périmètres environnants et les polluent par extension. On note aussi la présence de pneus usagés contenant des eaux usagées et qui sont des risques sanitaires importants.

Quant aux rares activités industrielles de la ville, leur incidence est ressentie sur l'environnement. L'activité industrielle locale comprend la brasserie de la Société de Limonaderies et Brasseries d'Afrique (SOLIBRA), les broyeuses de manioc, les scieries et les rizeries artisanales. A l'exception de la SOLIBRA située à la périphérie de la ville, sur la voie menant à Bonon et Daloa, les broyeuses de manioc, les scieries et les rizeries artisanales sont situées dans les quartiers. La transformation du manioc implique la présence de moulins dans ces quartiers. Les broyeuses produisent des déchets qui 
encombrent le sol. Les salissures sont composées des restes de manioc et des eaux usagées. Pour les besoins de cette activité, des fosses sont creusées pour recueillir les eaux provenant des broyeuses. Or ces fosses ne sont pas protégées si bien qu'une fois remplis d'eaux usagées, elles débordent en direction des pentes de terrain. Dans certains endroits, faute de fosses septiques, les eaux usages sont déversées dans les rues adjacentes. L'absence de caniveaux aggrave la situation sanitaire dans les quartiers qui sont alors envahis par les larves de moustiques vecteurs du paludisme.

Quant aux scieries, également génératrices de pollution, elles laissent dans les rues d'énormes sciures de bois qui encombrent les voies de circulation. Dans les périphéries des scieries, le sol est recouvert de sciures de bois provenant des activités des usines. Ces sciures inondent les rues et les habitations avoisinant les scieries. L'air ambiant a une odeur de bois mêlée de celle des produits de traitement du bois. Or, les sciures sont utilisées par les ménages comme combustible pour la cuisine, et il est fréquent d'en voir des stocks importants à l'intérieur des habitations. Ceux abandonnés sur la voie publique, se transforment compost (Photo 2).

Photo 2 : Une rue recouverte de compost dans un quartier de Bouaflé



Cliché : ADJELOU Kessou, Bouaflé, janvier 2017

Concernant les rizeries artisanales, les déchets qui se dégagent des moulins recouvrent le sol à l'instar des sciures de bois. Ces déchets sont stockés par endroits et ne sont jamais enlevés. Ce sont d'énormes tas de coques de riz qui finissent par devenir du compost. Plusieurs rues de Dioulabougou, de Biaka, de Datécouma et de Déhita sont impactées par ces résidus. Certes la brasserie de SOLIBRA dispose d'une décharge située sur la route menant à la localité de Blanfla, mais cette décharge comprend uniquement des déchets banals (cartons, boites, bouteilles). En plus, l'usine n'est pas dotée d'un centre de traitement des eaux usées. Cette situation s'explique certainement par la 
présence du fleuve Bandama, où les eaux usées de SOLIBRA sont dirigées, de même que les eaux usées de plusieurs activités économiques de la ville.

Enfin, l'activité principale à Bouaflé demeure l'agriculture, à cause des friches urbaines disséminées dans l'espace urbain, donnant l'impression d'être en zone rurale. Les alentours du canal de la rivière Gropéni sont particulièrement prisés par ces friches, où les ménages pratiquent la culture du maïs, du manioc, de la banane, de l'igname, ainsi que le tarot et les produits maraîchers. Dans certaines cours d'habitation, ces produits sont également cultivés, transformant ces espaces en des nids de moustiques et de bactéries. Certains ménages disposant d'animaux d'élevage, il n'est pas rare de rencontrer des chèvres, des lapins, des poulets, des bovins sur les mêmes espaces que les chiens et les chats. Sous ce rapport, les quartiers Dioulabougou, Déhita, Agbanou, Koblata, Marahoué, Hermankono apparaissent comme des fermes géantes. Cette situation pose le problème d'élimination des déchets.

Au total, les déterminants de la mortalité infanto-juvénile dans la ville, relèvent principalement de l'état d'insalubrité dans laquelle baigne la ville. En effet, Bouaflé est devenue une sorte de décharge à ciel ouvert, accueillant toutes sortes d'immondices dans l'espace urbain. Cependant, cette situation n'est pas propre à Bouaflé car, la plupart des villes ivoiriennes connaissent des problèmes similaires, y compris l'agglomération d'Abidjan, la capitale économique, et Yamoussoukro, la capitale administrative et politique du pays. Dès lors, se pose à l'échelle locale, la question de la gouvernance urbaine de la cité du Marahoué.

\section{Assainir l'environnement de la ville}

La gestion des déchets ménagers, une équation à géométrie variable

La gestion des déchets constitue l'un des grands défis de la gouvernance des Etats, à travers le monde. Par définition, cette expression désigne ce dont on veut se débarrasser. Le déchet implique donc un tri, un flux, et une sortie de son lieu de production pour être éliminé ou stocké (Goeury et Sierra, 2016 : 32). La réduction des risques de maladies dépend d'une gestion responsable des déchets, évitant la pollution de l'environnement. Or, les déchets solides ménagers sont les ordures les plus présentes à Bouaflé. Dans les quartiers d'habitation, les résidences et les commerces se côtoient, des restes de nourriture jonchent le sol des espaces partagés, alors que dans le quartier administratif, ce sont les feuilles de papier qui sont les plus visibles dans le paysage. Les difficultés des ménages commencent lorsqu'ils doivent se débarrasser ces ordures.

Les ménages évacuent les ordures dans les centres de collecte municipaux, mais aussi dans la brousse environnante, ou dans les dépôts sauvages créés volontairement dans les quartiers. Pourtant, le service 
technique de la mairie dispose de plusieurs sites de collecte d'ordures ménagères à travers la ville. Ces sites de regroupement autorisés sont équipés de bacs à ordures. Le service technique organise le transport des ordures, à partir des sites de regroupement vers la décharge publique, grâce à des tracteurs et des bennes à ordures. Pour mener efficacement cette tâche, les autorités municipales ont défini un circuit de ramassage des ordures qui couvrent les sites de collecte installés à travers la ville. Malgré les dispositions prises par les autorités municipales, les sites de regroupement sont délaissés au profit de la brousse environnante ou de sites de dépôts sauvages. Les services municipaux ignorent ces sites de dépôts qui changent continuellement, selon les difficultés rencontrées par les ménages. De ce fait, les agents de collecte ne visitent presque jamais ces sites où des ordures s'accumulent, atteignant parfois une hauteur impressionnante.

Finalement, à cause des difficultés éprouvées par les agents municipaux dans la collecte des ordures ménagères, Bouaflé offre l'image d'une ville poubelle où foisonnent des dépôts d'ordures. Notre enquête a identifié 133 différents dépotoirs, sur la voies menant vers la rivière Gropeni, de même, les ordures sont visibles à des carrefours, sur les voies non bitumées. Des décharges d'ordures sont érigées devant des cours d'habitation, des dépotoirs sont recensés le long de la rivière Gropeni traversant la ville, et des vidoirs entourent des aires de jeux et des écoles. Certains déchets datent de tellement longtemps que dans plusieurs endroits, ils se sont décomposés, devenant des matières organiques. Le compost qui est le résultat de la transformation chimique (Photo 3), est transporté par la rivière, à travers plusieurs quartiers (Agbanou, Dioulabougou, Koko-commerce) avant de se jeter dans le fleuve Marahoué. Or l'eau de cette rivière alimente, par arrosage, les friches urbaines dans le quartier Koko-commerce.

Photo 3 : Des déchets devenus du compost au quartier Agbanou

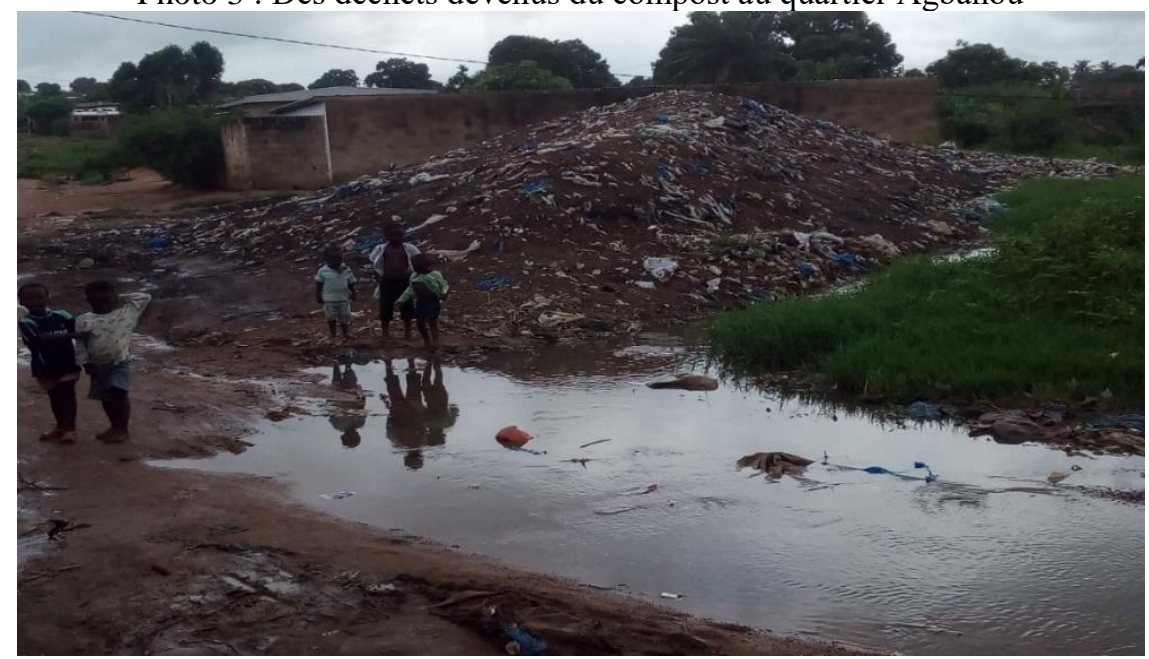

Cliché : ADJELOU Kessou, Bouaflé, décembre 2016 
Ces déchets stockés depuis longtemps dans les différents sites, apparaissent sous la forme d'un liquide noirâtre à la faveur des pluies. Connu sous le nom de lixiviat, ce liquide noirâtre et huileux traduit le degré de pollution de ces sites. Sa destination finale est le fleuve Marahoué qu'il pollue constamment.

Cette situation met à nu, l'impuissance des autorités municipales face à la dégradation de l'environnement local. Il est à noter que l'Etat ivoirien est centralisé à l'image de la France de la V ${ }^{\text {ème }}$ République dont il tire ses institutions. De ce fait, certaines décisions de gestion locale dépendent du pouvoir central d'Abidjan. Toutefois, des mesures administratives nationales concèdent certaines prérogatives aux autorités municipales, pour organiser la gestion des ordures ménagères en milieu urbain. Ainsi, les textes de l'organisation municipale de janvier 1978 (Ministère de l'Intérieur, 1978) et les lois et Décrets relatifs au mouvement communal ivoirien, entre 1980 et 1992, marquent la volonté étatique de décentraliser la gestion des ordures ménagères dans les collectivités territoriales.

Ces mesures indiquent notamment que jusqu'en 1995, des sociétés privées assurent une gestion déléguée des ordures ménagères (Société Industrielle de Transports Automobiles Africaines ou SITAF, ASH International). En outre, dès juillet 2003, la loi organise le transfert et la répartition des compétences de l'Etat aux collectivités territoriales (Ministère de l'Intérieur, juillet 2003), suivie en 2005, d'un texte relatif à la protection de l'environnement (RCI, Journal Officiel : 2006 : 31). La dégradation avancée de l'environnement des villes ivoiriennes, due à la décennie de crise sociopolitique (2000-2010), amène cependant l'exécutif ivoirien à la prise d'une Ordonnance en 2007 (Présidence de la République, 2007) abrogeant certaines dispositions de la loi de 7 juillet 2003, relative au transfert et à la répartition des compétences des collectivités territoriales. L'abrogation de ces dispositions ouvre la voie à une recentralisation de la gestion des ordures, grâce à la création, en 2007, d'un organisme public à caractère industriel et commerciale, l'Agence Nationale de la Salubrité Urbaine (ANASUR). Finalement, la gestion des ordures ménagères dans les villes ivoiriennes, dont Bouaflé, souffre de l'orthodoxie centralisatrice de l'Etat ivoirien, et ce, en dépit de toutes les mesures prises dans le cadre de la décentralisation. Pourtant, il faut résoudre les problèmes environnementaux qui impactent la qualité de la vie dans cette ville.

Résoudre les problèmes environnementaux

Les problèmes environnementaux à Bouaflé sont appréhendés selon deux logiques d'actions publiques. Premièrement, des actions ponctuelles de salubrité publique sont menées sur le terrain, initiées par les autorités municipales. Avec l'appui du Conseil Régional la municipalité décrète des opérations de salubrité, appelées " opération grand ménage », consistant au 
nettoyage des rues et des abords des habitats de la ville. En 2017, le lancement officiel de cette opération a eu lieu le 6 mai, au quartier Koko-commerce, en présence des autorités locales et des cadres de la région du Marahoué. Cette activité est souvent une occasion pour les autorités de faire des dons de matériels. A Bouaflé, «l'opération grand ménage » a été marquée, entre autres, par la destruction d'un immense dépôt sauvages d'ordures jouxtant l'école maternelle municipale de la ville.

A travers cette opération, la gouvernance locale marque son adhésion au programme gouvernemental de l'opération « grand ménage » initiée par le Ministère de la Salubrité, de l'Environnement et du Développement Durable (MINSEDD), dont Bouaflé a abrité la 4 édition en 2017. A cause de l'état de dégradation avancée de l'environnement local, Bouaflé est la première ville de l'intérieur du pays, à recevoir cette caravane de salubrité publique. L'objectif visé est d'exhorter les populations à la culture de la propreté, et à la promotion d'un modèle d'engagement citoyen, pour la propreté du cadre de vie en Côte d'Ivoire.

Dans le cadre de cette opération ponctuelle de lutte contre l'insalubrité dans la ville, le gouvernement ivoirien a fait un don d'équipements à la municipalité de Bouaflé, offrant deux camions bennes à ordures ménagères, cinq tricycles, ainsi que des kits de salubrité, par le biais du Conseil Régional et du Ministère de la Salubrité, de l'Environnement et du Développement Durable (MINSEDD). Ces dons traduisent une volonté des pouvoirs publics de responsabiliser les acteurs locaux avec des moyens adéquats, les invitant à mettre en place une brigade de salubrité composée de jeunes volontaires. La mission de ces équipes est de sensibiliser la population dans les quartiers. Les équipes constituées travaillent avec le Comité régional de salubrité et d'hygiène, présidé par le préfet de la ville.

Cependant, « l'opération grand ménage » présente des limites. Elle est ponctuelle et donc limitée dans le temps. En outre, sa mise en œuvre dépasse le cadre de la commune, puisque décidée et actée depuis le cabinet du Ministère de tutelle basé à Abidjan. Sous ce rapport, cette action de salubrité publique s'apparente à une opération de communication politique. Pour qu'elles soient profitables aux communes, les opérations de salubrité doivent être soutenues par des projets innovants et durables de salubrité publique. D'où la nécessité de prise en compte d'une seconde logique de l'action municipale, notamment celle qui place l'action publique en matière de salubrité dans une perspective de développement durable.

Dans cette dynamique, la mairie de Bouaflé a deux projets, dont l'un envisage de pré-collecter les ordures ménagère, en déposant des bacs à ordures dans certains endroits des quartiers, à recruter des pré-collecteurs qui passeront dans les ménages, en vue de recueillir les ordures et les acheminer vers ces bacs. Des coffres à ordures et des tracteurs de transport sont prévus, 
l'objectif visé par la municipalité étant de doter chaque quartier de camions de ramassage. Ces actions seront couplées avec celles visant l'éradication des dépôts sauvages grâce à un appel lancé à des entreprises privées.

C'est ici qu'intervient le deuxième projet de la ville, porté par le Conseil Régional, et qui vise la construction d'une usine de valorisation des ordures, en collaboration avec toutes les mairies de la région et la construction d'une unité de valorisation de troncs de bananier pour la fabrication de mèches, de papiers et de filasses pour des sacs biodégradables. L'intercommunalité de projet prôné par le Conseil Régional de Bouaflé est en cela innovant et porteur d'avenir, tel qu'analysé par divers travaux (CHICOT, 2016).

Concernant les activités économiques destructrices de l'environnement, la mairie a décidé de mettre fin au laxisme qui accompagne les activités des opérateurs économiques de la ville. Les scieries, les broyeuses de manioc, les rizeries, les garages automobiles et motocyclistes, les restaurateurs et les tenanciers de maquis seront désormais confrontés à la taxe pollueur-payeur. Les usines telles que les rizeries et les scieries seront délocalisées en dehors de la ville, pour y occuper des sites aménagés, comme c'est le cas de la brasserie locale de l'usine SOLIBRA. En tout état de cause, les opérateurs qui génèrent de la pollution et dont les activités dégradent l'environnement, doivent assumer leur part de coûts liés aux mesures de prévention, de réduction et de contrôle de la qualité de l'environnement. C'est ce qui ressort d'un entretien avec les responsables municipaux.

En outre, d'après la direction locale de l'environnement, des campagnes de sensibilisation de la population à la protection de la rivière « Gropéni » sont prévues. Principale source d'alimentation de la ville en eau potable, ce fleuve pourra être cédé aux entreprises de fabrication d'eau minérale, et d'aménagements de ses abords à des fins touristiques. L'ambition des autorités municipales étant de faire de Bouaflé, une ville durable, des espaces verts sont prévus dans les lotissements ultérieurs. Cette localité évoluant dans un environnement végétation de forêt, le projet vise la destruction des végétaux nocifs, s'appuyant sur l'urbanisme écologique auquel des ONG seront associées, dans la prise en compte simultanément, des enjeux sociaux, économiques, environnementaux et culturels de l'urbanisme, afin de mieux garantir les intérêts des collectifs citadins.

\section{Conclusion}

La mortalité infanto-juvénile, à Bouaflé, est causée par une série de pathologies parmi lesquelles l'anémie, les infections néonatales, la méningite, le paludisme, la pneumopathie et les souffrances cérébrales sont les plus fréquentes. Ces motifs de mortalité sont dominés par des maladies environnementales. L'état de l'environnement urbain constitue, par 
conséquent, un réel problème à Bouaflé. Le déficit en matière d'élimination des eaux usées, les difficultés de gestion des déchets solides issus des ménages et des activités économiques, et trop longtemps stockés dans les rues, impactent considérablement l'environnement de cette ville. Or, ces espaces jonchés de débris et de résidus en provenance de toutes parts, sont des terrains de jeux des enfants. En outre, la ville présent un déficit, en matière d'alimentation en eau potable. Pourtant, la ville durable comporte des enjeux à la fois sociaux, économiques et environnementaux, que la gouvernance locale est censé promouvoir, par des actions d'aménagement adéquat. C'est loin d'être le cas à Bouaké. En l'absence d'une politique cohérente en matière d'entretien de l'environnement local, une hypothèque pèse sur le devenir de Bouaflé dont la situation végétative des quartiers interpelle les autorités de la ville.

\section{References:}

1. BARBIERIE M., (1991), Les déterminants de la mortalité des enfants dans le tiers-monde, Paris, CEFEP, $46 \mathrm{p}$.

2. BLEY D., et BAUDOT P., (1997), «Les déterminants de la mortalité infantile dans la province de Marrakech $» I n$ : Conception, naissance et petite enfance au Maghreb, ${ }^{\circ}$ 9-10 : pp. 55-66.

3. BORDA P., (2016), «Territoire et développement ", in CHICOT P.Y. (dir.), Mutations des communes. Quelle intercommunalité de projets? Pour quel développement des territoires locaux? Editions L'Harmattan, Paris, p. 261- 267.

4. CHICOT, P.- Y., (dir.), (2016), Mutation des communes, Quelle intercommunalité de projets? Pour quel développement des territoires locaux? L'Harmattan, Paris, $443 \mathrm{p}$.

5. DIAKITE A., (2005), Les facteurs de mortalité néonatale infantile et juvénile dans la commune III du district de Bamako, Bamako, Faculté de Médecine de Pharmacie et d'Odontostomatologie, 63 p.

6. ESSO L., J. C. E., (Ed), (2013), Les déterminants de la mortalité des enfants de moins cinq ans en Côte d'Ivoire, Abidjan, Université Félix Houphouët-Boigny et Centre Suisse de Recherches Scientifiques en Côte d'Ivoire (CSRS), $150 \mathrm{p}$.

7. GOEURY D., SIERRA Ph., (2016), Introduction à l'analyse des territoires, Concept, outils, applications, Armand Colin, Paris, p. 3233.

8. KAÏD TLILANE N., (Ed), 2003, Les déterminants de la mortalité des enfants d'âge préscolaire en Algérie, Constantine, Laboratoire Economie et Développement, Université Abderrahmane Mira Bejaia, $102 \mathrm{p}$.

9. LACHAUD J.-P., (2002), Modélisation des déterminants de la 
mortalité des enfants et pauvreté aux Comores, Bordeaux, Université Montesquieu-Bordeaux IV, $31 \mathrm{p}$.

10. NGUESSAN L. D. et al, (1990), La mortalité infantile et ses causes dans une sous- préfecture de Côte d'Ivoire, Abidjan, revue Médecine tropicale, $432 \mathrm{p}$.

11. NIEL X., (2013), Les facteurs explicatifs de la mortalité infantile en France et leur évolution récente, Paris, INSEE, $35 \mathrm{p}$.

12. PACE R., et MASTROROCCO N., (2001), Déterminants de la mortalité infantile à travers quelques parcours causaux, Bari, Université de Bari, pp 779-789.

13. GUILLEMOT L., (2015), «Temporalités des espaces publics centraux. » In SOUMAGNE J., (dir.), Temps et usages de la ville. Presses Universitaires de Rennes, 248 p. 